Quota per capita de água, fatores intervenientes e modelagem: estudo de caso para classes socioeconômicas de Cuiabá-MT Welitom Ttatom Pereira da Silva, Aldecy Almeida Santos, Luiz Airton Gomes, Carlo Ralph de Musis

\title{
QUOTA PER CAPITA DE ÁGUA, FATORES INTERVENIENTES E MODELAGEM: ESTUDO DE CASO PARA CLASSES SOCIOECONÔMICAS DE CUIABÁ-MT
}

\section{Water per capita quota, influence factors and modeling: a case study to socio-economic class of Cuiaba-MT}

\author{
Welitom Ttatom Pereira da Silva \\ Doutorando em Tecnologia Ambiental e Recursos Hídricos pela Universidade de Brasília \\ ttatom@universiabrasil.net \\ Aldecy Almeida Santos \\ Mestrando em Física e Meio Ambiente pela Universidade Federal de Mato Grosso \\ aldecyalmeida@ufmt.br \\ Luiz Airton Gomes \\ Professor Dr. da Faculdade de Arquitetura, Engenharia e Tecnologia da Universidade Federal de Mato Grosso \\ la.gomes@terra.com.br \\ Carlo Ralph de Musis \\ carlo@unic.br
}

Professor Dr. do Programa de Estudos Pós-Graduados em Física Ambiental da Universidade Federal de Mato Grosso

Artigo recebido para publicação em 19/03/08 e aceito para publicação em 01/10/08

RESUMO: O presente trabalho tem por objetivo estimar modelos matemáticos que projetem quotas per capita de água em cada classe de rendimento (alta, médio-alta, média, médio-baixa, baixa) a partir de variáveis intervenientes no município de Cuiabá-MT. Os resultados indicaram a variável econômica como variável explicativa (tarifa da água), e as climáticas, pouco relevantes ao estudo. Foram propostos modelos matemáticos à projeção de quota per capita de água para as classes de rendimento alta e médio-alta. As baixas correlações entre a quota per capita e variáveis dependentes não permitiram a estimativa de modelos matemáticos às classes de rendimento média, médio-baixa e baixa.Palavras-chave: modelagem, Cuiabá, classe de rendimento, recursos hídricos.

ABSTRACT: The present work has the main aim to estimate mathematical models which project per capita quota of water in the various classes of income groups (high, medium high, medim, medium low, low) from the point of view of interfering variables in the Cuiaba municipality, MT. The results indicated that climatic factors have low impact. Here are proposed mathematical models indicating projection of per capita quota of water for the different classes of income, such as high and medium high. The poor correlations between the per capita quota of medium, low-medium and low income groups do not permit the estimate of mathematical models.Keywords: Mathematical model, Cuiaba, classes of income, hydrological resources. 


\section{INTRODUÇÃO}

A cidade de Cuiabá está localizada na região centro-sul do estado de Mato Grosso e apresenta-se hoje como um dos pólos de desenvolvimento da região Centro-oeste do Brasil. Esta possui atualmente uma das maiores taxas de crescimento populacional do país, 2,77\% a.a. (CUIABÁ, 2004). Também se registrou taxas de crescimento industrial e comercial de 17 e $21 \%$, respectivamente, (FIEMT, 2005). Associado a este crescimento encontra-se a necessidade de atendimento à demanda por infraestrutura, fator intimamente relacionado ao consumo de recursos naturais. Daí a imperativa obrigação de gestão dos recursos naturais. Dentro desse contexto, encontra-se a gestão dos recursos hídricos que visa, essencialmente, melhorar a qualidade da água de maneira a viabilizar seus múltiplos usos (Amaral e Shirota, 2002).

A gestão de recursos hídricos, em sentido lato, é a forma pela qual se pretende equacionar e resolver as questões de escassez relativa dos recursos hídricos, bem como fazer o uso adequado, visando a otimização dos mesmos em benefício da sociedade. Segundo Setti et al. (2001) a implementação dessa gestão concretizase por um conjunto de ações de planejamento; de elaboração, de regulamentação e aplicação de leis; de incentivo econômico à gestão financeira, e do inventariado dos recursos hídricos. Logo, informações relativas à quota per capita de água (QPCA) podem ser consideradas ferramentas de grande valia quando se deseja crescimento urbano sustentável. A previsão da QPCA é um instrumento básico de planejamento, necessário para o correto dimensionamento da oferta e para o direcionamento de medidas de gestão da demanda. Fundamentalmente esta previsão consiste na projeção de comportamentos observados no passado, considerando os fatores que possam alterar as tendências futuras. Embora simples em sua formulação e auto-explicativa em sua necessidade, a previsão da demanda não tem sido um instrumento utilizado no Brasil (Silva e Rocha, 1999). O próprio indicador de QPCA é sujeito a variações importantes no tempo, especialmente em contextos, como o brasileiro, onde se verificam expressivas taxas de expansão e adensamento em áreas específicas da cidade (Silva e Rocha, 1999). A utilização na elaboração de projetos ambientais de valores de QPCA, tabelados, ou de cidades com características semelhantes, tem sua validade questionável na previsão de demandas reais dada a co-variação desta numa comunidade com fatores como nível sócioeconômico, industrialização, clima, porte, topografia, percentual de hidrometração, custo da tarifa, entre outros (Fernandes Neto et al, 2004).

Campos e von Sperling (apud Fernandes Neto et al., 2004, p.102) relacionaram classe econômica (alta, média alta, média, média baixa e baixa) e QPCA, em um estudo realizado em nove bairros de Belo Horizonte e Contagem-MG. Os resultados apresentaram correções bastante significativas entre a QPCA e a renda per capita $(\mathrm{r}=0,942)$, a área do lote $(\mathrm{r}=0,887)$ e o número de vasos sanitários $(\mathrm{r}=$ $0,810)$, respectivamente.

Zhou et al. (apud Fernandes Neto et al., 2004, p. 102) avaliam os efeitos da temperatura na QPCA da cidade australiana de Melbourne. Detectou-se uma relação complexa entre estas duas variáveis, com duas regressões representando o fenômeno.

Amaral e Shirota (2002) estudaram os efeitos dos componentes sazonais e o ciclo de tendência do consumo de água que estimaram a média de consumo de água para a cidade de Piracicaba-SP. Os resultados mostraram que a QPCA apresentava forte componente sazonal, e que esta era diretamente proporcional à temperatura.

Borges (2003) realizou um estudo intitulado “Acompanhamento de um modelo de previsão de demanda de água a um modelo de simulador em tempo real - estudo de caso: sistema adutor metropolitano de São Paulo", em que foi proposta uma evolução metodológica na evolução do sistema operacional, via implantação de um modelo matemático, em tempo real, de previsão do consumo de água horário. Os resultados indicaram a possibilidade de estabelecimento de regras operacionais mais eficientes. 
Trautwein (2004) em "Avaliação de métodos para previsão do consumo de água para curtíssimo prazo: um estudo de caso em empresa de saneamento" analisou dois métodos de previsão de consumo de água, o método do ajuste exponencial e o método das redes neurais. Os resultados da pesquisa comprovam a eficiência das previsões de consumo em intervalos de hora, mas não mostram a existência de um modelo ideal, que possa ser aplicável em qualquer sistema de abastecimento de água.

Considerando a escassez e a necessidade de informações à adequada gestão dos recursos hídricos, este trabalho tem por objetivo estimar modelos matemáticos que projetem QPCA em cada classe de sócio-econômica (alta, médio-alta, média, médiobaixa, baixa), a partir de variáveis intervenientes no município de Cuiabá-MT.

\section{CARACTERIZAÇÃO DA ÁREA DE ESTUDO}

O município de Cuiabá possui área de $3.538,17 \mathrm{~km}^{2}$, correspondendo $254,57 \mathrm{~km}^{2}$ à Macrozona Urbana e $3.283,60 \mathrm{~km}^{2}$ à Área Rural. Localiza-se na mesorregião Norte-Matogrossense, nas coordenadas geográficas $15^{\circ} 35^{\prime} 56^{\prime \prime}$ de latitude sul (S) e $56^{\circ} 06^{\prime} 01^{\prime \prime}$ de longitude Oeste (W) de Greenwich (Gr).

Situa-se na província geomorfológica denominada Baixada Cuiabana. Esta consiste numa peneplanície de erosão, onde predominam relevos de baixas amplitudes e altitudes variam de 146 a 250 metros (IDPU, 2006).

O clima é essencialmente Tropical Continental, mas com algumas variantes típicas do lugar, apresentando dois períodos distintos: o chuvoso, com duração de oito meses, e o seco, com duração de quatro. No período chuvoso, principalmente nos primeiros meses, a temperatura é mais elevada. Geralmente o período das chuvas começa e termina por ventanias violentas acompanhadas de trovões. Nessa época do ano a pressão atmosférica, mantendo-se alta, permite suportar as temperaturas elevadas. A umidade do ar mantém-se alta. Assim, durante a época das chuvas, o clima é Tropical Continental Úmido. O período seco estende-se de junho a setembro, havendo alguns anos com períodos mais pronunciados. A umidade do ar em certos dias desce a uma percentagem mínima que varia de $18 \%$ a $40 \%$. Essas características definem o clima como Tropical Continental Seco, durante esses meses. É no período seco que ocorrem as frentes frias, ocasionadas pelo avanço das massas de ar vindas dos pampas argentinos, situados mais próximos das geleiras do Pólo Sul. O frio geralmente acompanhase de garoas persistentes empurradas para o norte por ventos intensos do sul; permanece, no máximo, oito dias, para reaparecer a intervalos mais ou menos prolongados (CUIABÁ, 2007).

A cidade é abundante em recursos hídricos: diversos rios, ribeirões e córregos formadores da bacia do rio Cuiabá banham-na. A acelerada urbanização e o crescimento econômico por que passou Cuiabá a partir dos anos 70 alcança e afeta também o rio, parte integrante da cidade. Principal recurso hídrico teve intensificado e diversificada sua utilização, o que aumentou a captação de suas águas (CUIABÁ, 2004). A Companhia de Saneamento da Capital (Sanecap) é a atual instituição gestora dos recursos hídricos em Cuiabá, Cuiabá (2004). Atualmente a mesma apresenta os seguintes indicadores do sistema de abastecimento de água: cobertura da cidade 97\%; extensão de rede $2.120 \mathrm{Km}$; adutora de água tratada $63,35 \mathrm{Km}$; adutora de água bruta $23,87 \mathrm{Km}$; total de ligações de água 133.383; total de economias 168.814; volume faturado $2.693 .551 \mathrm{~m}^{3} / \mathrm{mês}$; índice de hidrometração $62,81 \%$; perda total 45 - 50\%; evasão de receita 17,65\%; arrecadação de cerca de R\$ 4.588.334,00 para o ano de 2006. Também se registra nove sistemas de tratamento de água distribuídos entre as áreas urbana e rural, com vazões de tratamento variando entre 226 a $4270 \mathrm{~m}^{3} / \mathrm{h}$ (SANECAP, 2007). 


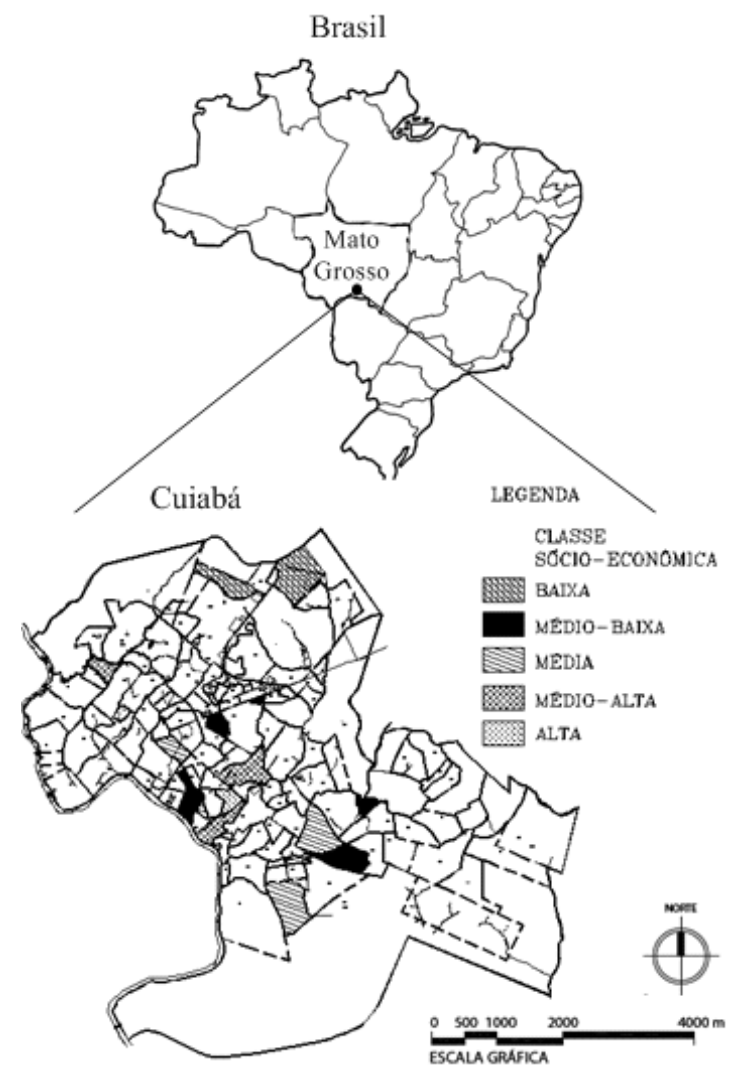

Figura 1. Mapa de Cuiabá e bairros inseridos no universo amostral.

Na Figura 1, tem-se apresentado a localização de Cuiabá, um mapa com bairros selecionados aleatoriamente, e as suas respectivas classes sócioeconômicas de cada bairro.

\section{METODOLOGIA}

Como método amostral, assumiu-se como metodologia de trabalho a amostragem aleatória estratificada, onde se definiu como população o agrupamento de bairros, conforme classes de rendimento (CR), conforme dados do CUIABÁ (2004); o estrato amostral como o bairro; e a unidade amostral sendo o valor observado em cada mês estudado. A média aritmética da renda por classes é apresentada na Tabela 1.

Tabela 1 - Renda média aritmética por classe econômica.

\begin{tabular}{c|c}
\hline CLASSE & LIMITES \\
\hline Renda média de Cuiabá & 7,49 S.M. \\
\hline Renda Baixa & Abaixo de 2,91 S.M. \\
\hline Renda Médio-Baixa & De 2,91 a 5,65 S.M. \\
\hline Renda Média & De 5,66 a 11,65 S.M. \\
\hline Renda Médio-Alta & De 11,66 a 21,94 S.M. \\
\hline Renda Alta & Acima de 21,94 S.M. \\
\hline S.M.: salário mínimo & \\
\hline
\end{tabular}

Fonte: CUIABÁ (2004) 
A amostra piloto foi definida como sendo o número de unidades amostrais de estudo $(n)$, fixada inicialmente em $10 \%$ do total de bairros existentes em cada CR. Posteriormente, este número foi testado pelo cálculo do tamanho da amostra aleatória para estimar a média de uma população finita, conforme Martins (2002).

Quanto às variáveis de interesse que influenciam na QPCA, optou-se pelo emprego de algumas variáveis listadas por CETESB (1978) e von Sperling (apud Fernandes Neto et al., 2004, p. 102). Entre elas, a temperatura média mensal, média mensal da umidade relativa do ar, o índice pluviométrico e a tarifa cobrada ao consumidor.
Conforme Yassuda et al. (1976), diversos são os fatores que influenciam no consumo de água em cidades, dentre os quais: (i) o clima - o consumo aumenta conforme aumenta a temperatura; a umidade também exerce influência, dado que em regiões mais secas o consumo é maior; a presença de chuvas afeta diretamente o consumo, reduzindo-o drasticamente na sua ocorrência; (ii) o preço do serviço - o preço elevado das tarifas de água e esgoto promove a redução do consumo de água, interferindo diretamente nos hábitos da população; (iii) nível sócio-econômico da população.

As variáveis estudadas, sua codificação (Cod.), suas unidades e fontes de dados são apresentadas na Tabela 2.

Tabela 2 - Variáveis estudadas e fontes de dados.

\begin{tabular}{l|l|l}
\hline VARIÁVEL & UNIDADES & \multicolumn{1}{c}{ FONTES DOS DADOS } \\
\hline $\begin{array}{l}\text { Índice Pluviométrico } \\
\text { (Cod.: INPL) }\end{array}$ & $\mathrm{mm} / \mathrm{h}$ & Instituto Nacional de Meteorologia (INMET) \\
\hline $\begin{array}{l}\text { Temperatura média mensal } \\
\text { (Cod.: TEMP) }\end{array}$ & ${ }^{\circ} \mathrm{C}$ & Instituto Nacional de Meteorologia (INMET) \\
\hline $\begin{array}{l}\text { Umidade média mensal } \\
\text { (Cod.: UMRE) }\end{array}$ & $\%$ & Instituto Nacional de Meteorologia (INMET) \\
\hline $\begin{array}{l}\text { Quota } \text { per capita de água } \\
\text { (Cod.: QPCA) }\end{array}$ & $\mathrm{L} / \mathrm{hab.dia}$ & $\begin{array}{l}\text { Agência de Saneamento da Capital (Sanecap) e } \\
\text { Instituto de Pesquisa e Desenvolvimento Urbano de } \\
\text { Cuiabá (IPDU-Cuiabá) }\end{array}$ \\
\hline $\begin{array}{l}\text { Tarifa de cobrança da água } \\
\text { (Cod.: TARI) }\end{array}$ & $\mathrm{R} \$ / \mathrm{m}^{3}$ & \begin{tabular}{l} 
Agência de Saneamento da Capital (Sanecap) \\
\hline
\end{tabular}
\end{tabular}

Os dados referem-se a uma série histórica de 6 anos, cujo período abrange de 2001 a 2006.

De posse do banco de dados, iniciaram-se os procedimentos analíticos estatísticos utilizando o software SPSS (Statistical Package for the Social Sciences). As análises estatísticas seguiram a seqüência apresentada na Figura 2.

Em relação à análise de regressão, prioritariamente, esta é usada com o propósito de se prever valores de uma variável (variável dependente) em função de valores possivelmente intervenientes (variáveis independentes). 


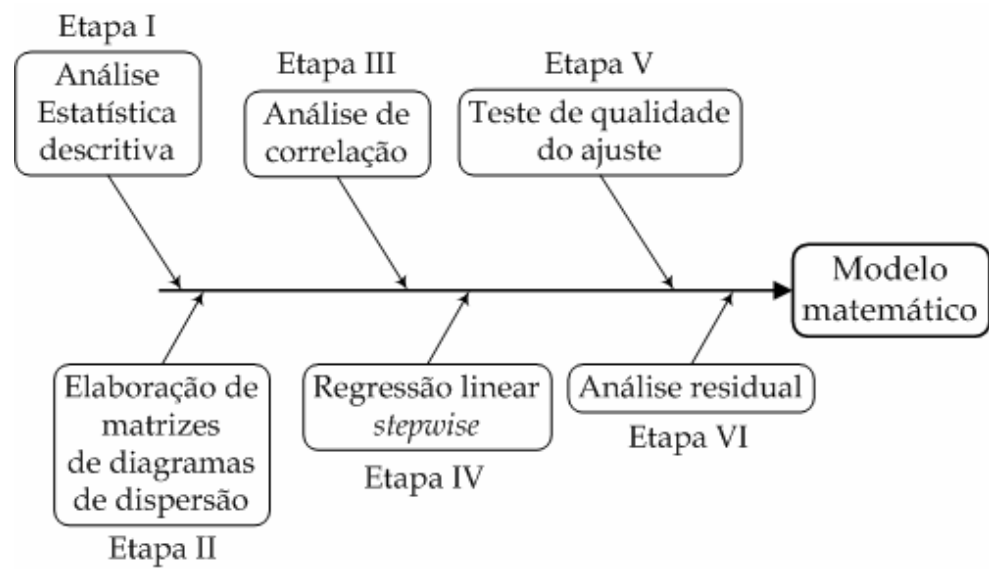

Figura 2. Fluxograma demonstrando as etapas da técnica de modelagem adotada.

O modelo de regressão linear múltipla é exemplificado na Equação 1.

$\mathrm{QPCA}=\beta_{0}+\beta_{1} . \mathrm{INPL}+\beta_{2} . \mathrm{TEMP}+\beta_{3} . \mathrm{UMRE}+$ $\beta_{4}$. TARI (Eq. 1)

Onde:

QPCA = quota per capita de água (L/hab.dia);

$\beta_{0}, \ldots, \beta_{4}=$ coeficientes de regressão;

INPL = índice pluviométrico $(\mathrm{mm} / \mathrm{h})$;

TEMP $=$ temperatura média mensal $\left({ }^{\circ} \mathrm{C}\right)$;

$\mathrm{UMRE}=$ umidade relativa média mensal (\%);

$\mathrm{TARI}=$ tarifa de cobrança da água $\left(\mathrm{R} \$ / \mathrm{m}^{3}\right)$.

\section{RESULTADOS E DISCUSSÃO}

Em relação ao universo amostral, verificouse que este foi satisfatório. Os resultados apresentaram como $n$ ideal de 58,48,65, 78 e 23 , e a amostrapiloto foi de 189, 441, 189, 189 e 63 para as classes de rendimento baixa, médio-baixa, média, médio-alta e alta, respectivamente.

Para cada uma das cinco $\mathrm{CR}$ foram realizadas análises estatísticas descritivas, conforme exemplificado na Tabela 3. A partir destas, constatouse valores crescente de desvio padrão na seguinte ordem de CR: 1, alta; 2, médio-baixa; 3, baixa; 4, média; e 5 , médio-alta.

Tabela 3. Análise estatística descritiva $\mathrm{CR}$ alta.

\begin{tabular}{l|l|l|l|l|l}
\hline & N & Mín. & Máx. & Média & Desvio Padrão \\
\hline INPL & 63 & 0 & 356 & 107,43 & 89,431 \\
\hline TEMP & 63 & 18,1 & 29,4 & 26,003 & 2,1026 \\
\hline UMRE & 63 & 52,0 & 96,0 & 71,937 & 9,8552 \\
\hline QPCA & 63 & 213 & 343 & 266,63 & 25,274 \\
\hline TARI & 63 & 2,80 & 3,70 & 2,8571 & 0,22122 \\
\hline
\end{tabular}

Fato contraditório, visto que demonstra que a classe alta tem comportamento aparentemente semelhante às classes médio-baixa e baixa, com relação ao estudo da QPCA. Também pode-se constatar valores médios de QPCA (L/hab.dia) de 266, 272, 172, 132 e 116, para as CR alta, médio-alta, média, médio-baixa e baixa, respectivamente. Valores estes que confirmam o preconizado em literatura: a existência da relação entre classe sócio-econômica e QPCA, além de confirmar a importância do nível de renda como fator interveniente. Os gráficos de dispersão não mostraram associação entre a QPCA e 
as variáveis de índice pluviométrico, tarifa e baixa e baixa, conforme exemplificado na Figura 3. temperatura, ao se observar os bairros de CR médio-

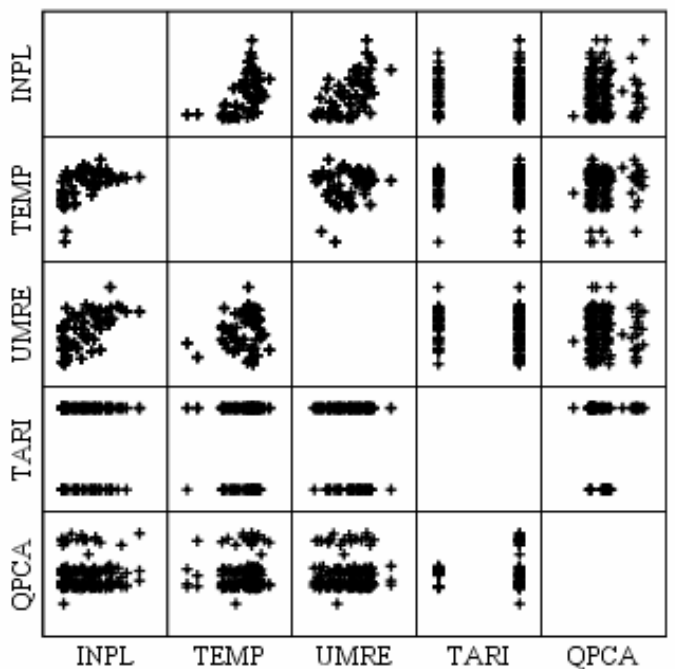

Figura 3. Matriz de diagrama de dispersão considerando QPCA, TARI, UMRE, INPL, para a CR baixa.

No entanto, indicaram a existência de uma associação entre a QPCA e a variável umidade relativa do ar, nos bairros de CR alta, médio-alta e média, conforme exemplificado na Figura 4.

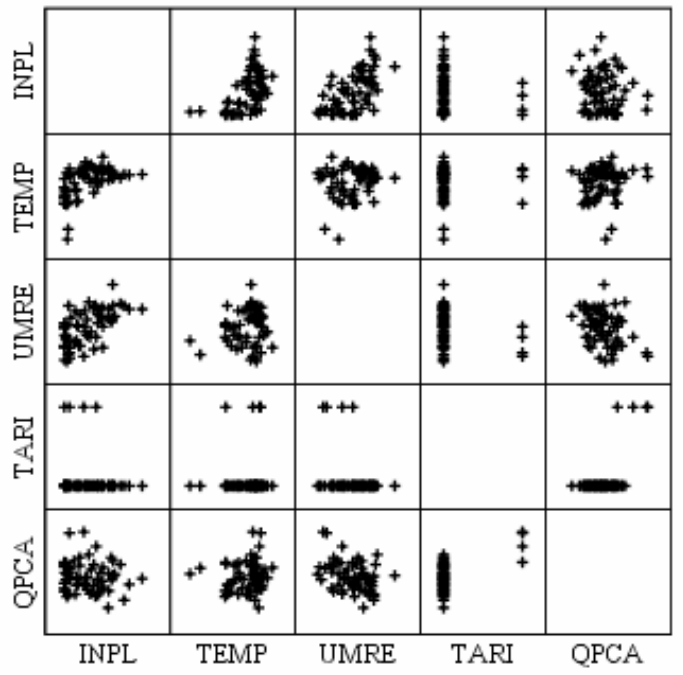

Figura 4. Matriz de diagrama de dispersão considerando QPCA, TARI, UMRE, INPL, para a CR alta.

Outro ponto observado foi o tipo de associação que, devido ao comportamento da nuvem, evidenciou dependência linear inversa ou associação negativa (Bussab e Morettin, 2006). Em outras palavras, quanto maior a UMRE, menores serão os valores de QPCA, nas CR alta, médio-alta e média.
Observou-se ainda associações entre as variáveis climáticas, associações já esperadas uma vez que se tem conhecida a existência da relação entre chuva, umidade e temperatura do ar. Em relação à análise de correlação deparou-se com valores considerados altamente significativos (nível significância de 0,01) 
entre as variáveis de QPCA, umidade relativa do ar e tarifa, para as CR alta e médio-alta. Quando observado o comportamento ao longo das CR, os coeficientes de correlação entre QPCA e INPL apontaram para uma mudança no tipo de correlação.

Por outro lado, notou-se que esta mudança era da correlação nula para a negativa, à medida que se caminhava no sentido crescente das $\mathrm{CR}$, conforme Tabela 4. Daí, quanto maior a CR, maior a intensidade de reação a esta condição climática. Contudo, devido aos seus baixos valores (coeficiente de correlação < $0,7)$ pode-se inferir que não havia forte correlação linear entre a variável QPCA e o INPL para o universo amostral.

Tabela 4. Correlação entre índice pluviométrico e CR.

\begin{tabular}{c|c}
\hline Classe de Rendimento & Coeficiente de correlação \\
\hline Baixa & 0,002 \\
\hline Médio-baixa & 0,013 \\
\hline Média & $-0,003$ \\
\hline Médio-alta & $-0,041$ \\
\hline Alta & $-0,134$ \\
\hline
\end{tabular}

Para o coeficiente de correlação da QPCA e TEMP encontrou-se o valor de 0,088 para CR alta, considerado baixo conforme literatura. Daí pode-se inferir que a temperatura não apresentou correlação na PQCA. Por este fato, a situação estudada diverge dos resultados encontrados por Amaral e Shirota (2002) que mostraram que o consumo de água apresenta um forte componente sazonal diretamente proporcional à temperatura. Uma possível explicação para este fato provavelmente está relacionada com a localização das duas áreas estudadas. A região de Cuiabá apresenta uma pequena variação nas temperaturas médias das mínimas e por um período muito curto; enquanto no segundo estudo, realizado para o Estado de São Paulo, estas mínimas eram mais pronunciadas. Quando se observou esta correlação ao longo das $\mathrm{CR}$, deparou-se com coeficientes de correlação variando de 0,088 e - 0,025, conforme Tabela 5.

Tabela 5. Correlação entre temperatura e CR.

\begin{tabular}{c|c}
\hline Classe de Rendimento & Coeficiente de correlação \\
\hline Baixa & 0,047 \\
\hline Médio-baixa & 0,008 \\
\hline Média & $-0,025$ \\
\hline Médio-alta & 0,066 \\
\hline Alta & 0,088 \\
\hline
\end{tabular}

Quanto aos modelos matemáticos de projeção da QPCA por CR, realizaram-se por meio de análise de regressão múltipla stepwise, tendo-se tomado as variáveis de INPL, UMRE, TEMP e TARI como variáveis explicativas. Foram utilizadas 1071 observações distribuídas nas CR.

Os resultados obtidos indicaram apenas dois modelos matemáticos. Estes resultados não eram esperados, uma vez que o objetivo era encontrar cinco 
Em relação à Estatística $F$, esta se mostrou altamente significativa, indicando que as variáveis explicativas são, conjuntamente, expressivas para explicar o comportamento da quantidade consumida de água, também em ambos os modelos.

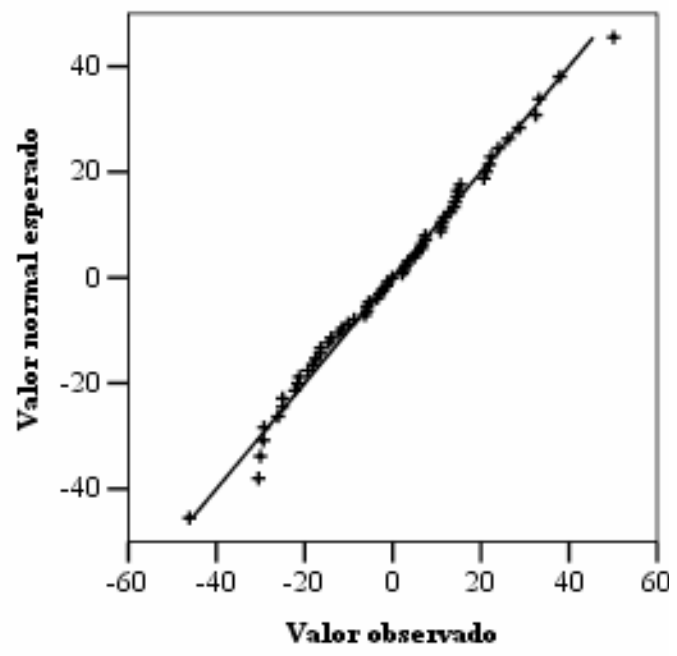

Figura 5. Gráfico Qq-plots de normalidade dos resíduos, modelo 1.

Analisou-se o comportamento dos resíduos dos modelos usando o conjunto de dados observados e estimados. No que diz respeito ao modelo 1, tem-se uma representação gráfica dos resíduos o gráfico $Q q$ plots de normalidade apresentados na Figura 5.

As representações gráficas dos resíduos mostram a existência de elementos discrepantes. Em relação à distribuição normal, verificou-se que os resíduos não eram normalmente distribuídos, uma vez que havia valores afastados da reta de normalidade. Daí sugere-se, a fim de se proceder à adequação do modelo matemático, a realização de estudos dos elementos discrepantes.

O modelo 2 apresentou como resultados de análise residual gráfica as Figura 6.

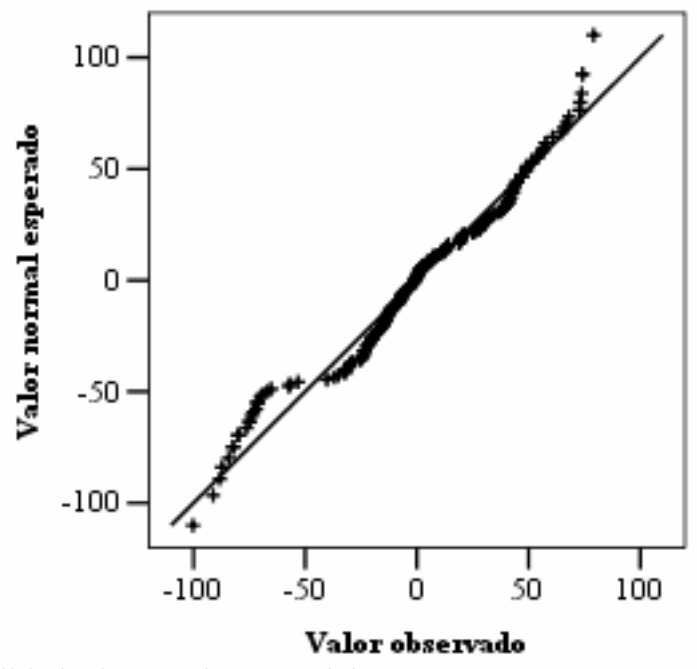

Figura 6. Gráfico Qq-plots normalidade dos resíduos, modelo 2. 
O gráfico dos resíduos do modelo 2, nos indica que temos possíveis inadequações do modelo adotado, e as curvas sugerem que se deve estudar outras funções matemáticas que expliquem melhor o fenômeno (Bussab e Morettin, 2006). A análise do gráfico Qq-plots de normalidade nos diz que a hipótese de normalidade não foi aceita, uma vez que se os resíduos fossem normais os pontos deveriam se dispor ao longo de uma reta. Tal fato indica que os modelos propostos foram considerados pouco competentes à projeção da QPCA de água. Uma possibilidade de justificativa seria a existência de variáveis intervenientes não consideradas no estudo, por exemplo, o percentual de hidrometração, a existência de rede coletora de esgotos e as características topográficas regionais.

\section{CONCLUSÃO}

Os resultados da análise descritiva confirmaram a relevância da classe socioeconômica para com a quota per capita de água. As QPCA foram 266, 272, 172, 132 e 116 L/hab.dia, para as CR alta, médio-alta, média, médio-baixa e baixa, respectivamente.

As análises de associação indicaram a variável econômica como variável explicativa (tarifa da água) e as climáticas, pouco relevantes ao estudo. Uma possível justificativa poderia ser a existência de variantes climáticas típicas do local (sazonalidade), apresentada por dois períodos distintos: o chuvoso, com duração de oito meses e o seco, com duração de quatro, conforme CUIABÁ (2004). Uma outra justificativa poderia estar no fato da existência de descontinuidade do serviço de fornecimento de água, o que restringiria o consumo em função da capacidade de reservação de cada residência.

Foram estimados modelos matemáticos à projeção de QPCA para as CR alta e médio-alta, no entanto, o banco de dados utilizado não possibilitou a validação de um modelo matemático que explicasse, de modo satisfatório, o fenômeno. As baixas correlações entre a QPCA e variáveis dependentes não permitiram a estimativa de modelos matemáticos.
Sendo, então, sugerida a adição de variáveis intervenientes não consideradas e a investigação dos elementos discrepantes.

\section{REFERÊNCIAS BIBLIOGRÁFICAS}

AGENCIA DE SANEAMENTO DA CAPITAL SANECAP. Consulta a indicadores do sistema de abastecimento de água. Disponível em: < https:// sanecap.locaweb.com.br/produtos.php? idcategoria=282 $>$ Acesso em: 14 abr. 2007.

AMARAL, A.M.P.; SHIRITA, R. Consumo residencial médio de água tratada: uma aplicação de modelos de séries temporais em Piracicaba. Revista Agrícola, v. 49, n. 1, p. 55-72, 2000.

BORGES, V.M.N.AAcompanhamento de um modelo de previsão de demanda de água a um modelo simulador em tempo real - um estudo de caso: sistema adutor metropolitano de São Paulo. 2003. 206 f. Dissertação (Mestrado em Engenharia Hidráulica) Universidade de São Paulo, São Paulo-SP.

BUSSAB, W.O.; MORETTIN, P.A. Estatística básica. 5. ed. São Paulo: Saraiva, 2006.

COMPANHIA DE TECNOLOGIA DE SANEAMENTO AMBIENTAL - CETESB. Técnica de abastecimento e tratamento de água. São Paulo: CETESB, 1978.

CUIABÁ. PREFEITURA MUNICIPAL. INSTITUTO DE PESQUISA E DESENVOLVIMENTO URBANO - IPDU. Perfil Socioeconômico de Cuiabá - Vol. II - Cuiabá: IPDU/AS\&M/Central de Texto, 2004. 405 p.

CUIABÁ. PREFEITURA MUNICIPAL. INSTITUTO DE PESQUISA E DESENVOLVIMENTO URBANO - IPDU. Perfil Socioeconômico de Cuiabá - Vol. III - Cuiabá: Central de Texto, 2007. 477 p.

FEDERAÇÃO DAS INDÚSTRIAS DO ESTADO DE MATO GROSSO - FIEMT. Análise de alguns 
indicadores macroeconômicos. Cuiabá, 2005. Disponível em: <http://www.fiemt.com.br>.Acesso em: 08 de abr. 2006.

FERNADES NETO, M.L. et al. Avaliação da relevância dos parâmetros intervenientes no consumo per capita de água para os municípios de Minas Gerais. $A B E S$, v. 9, n. 2, p. 100-107, 2004.

INSTITUTO NACIONAL DE METEROLOGICO INMET. Relatório climatológico. Várzea Grande: INMET, 2006. 4 p. (Relatório técnico, Rt-2001-2006).

MARTINS, G.A. Estatística geral e aplicada. 2. ed. São Paulo: Atlas, 2002.

SETTI, A.A. et al. Introdução ao gerenciamento de recursos hídricos. Brasília: Agencia Nacional de Energia Elétrica; Agência Nacional de Águas, 2001.

SILVA, I. Re: Informações de bairros - relatório Sanecap. Mensagem recebida por: $<$ ttatom@universiabrasil.net> em: 17 de nov. 2006.

SILVA, R.T.; ROCHA, W.S. Caracterização da demanda urbana de água. Brasília: Secretaria Especial de Desenvolvimento Urbano, 1999.

TRAUTWEIN JR, B. Avaliação de métodos para previsão de consumo de água para curtíssimo prazo: um estudo de caso para a empresa de saneamento. 2004. 107 p. Dissertação (Mestrado em Engenharia de Produção e Sistemas) - Pontifícia Universidade Católica do Paraná, Curitiba-PR.

YASSUDA E.R.; OLIVEIRA W.E.; GAGLIANONE S.; NOGAMI P.S.; PEREIRA B.E.B.; MARTINS J. A. Técnica de abastecimento e tratamento de águavol. 1. 2a. ed. São Paulo: CETESB, 1976. 